\title{
A case of myopericarditis caused by Neisseria meningitidis W135 serogroup with protracted inflammatory syndrome
}

\author{
Authors: Alex J Keeley, ${ }^{A}$ Daniel Hammersley ${ }^{B}$ and Sukhbir S Dhamrait ${ }^{C}$
}

Meningococcal pericarditis is classically divided into three separate entities: isolated meningococcal pericarditis, disseminated meningococcal disease with pericarditis, and reactive (immunopathic) meningococcal pericarditis. We present the case of a 74-year-old woman with meningococcal septicaemia with meningococcal myopericarditis, which demonstrates crossover features.

\section{Case presentation}

A 74-year-old caucasian woman with no history of immunosuppression or rheumatological disease, but with a history of paroxysmal atrial fibrillation (AF) for which she was taking flecainide but no anticoagulation, was admitted following a Baltic cruise holiday. She had fever, rigors, chest pain radiating to the back and neck, and progressive breathlessness of several days onset. On initial examination, she was in respiratory distress with a respiratory rate of 24 breaths/minute, oxygen saturations of $92 \%$ on $28 \%$ oxygen, tachycardia of 146 beats/minute, blood pressure of $133 / 73 \mathrm{mmHg}$ and a temperature of $37.5^{\circ} \mathrm{C}$. She had no peripheral oedema, a normal jugular venous pressure, normal heart sounds and bilateral inspiratory crepitations. There was no rash. Her 12-lead electrocardiogram showed AF with rapid ventricular response and subtle widespread $1 \mathrm{~mm}$ ST segment elevation, with ST segment depression in leads aVR and V1 (Fig 1). She had raised inflammatory markers with a white cell count of $12.3 \times 10^{9} / \mathrm{L}$ and C-reactive protein of $471 \mathrm{mg} / \mathrm{L}$, low sodium of $124 \mathrm{mmol} / \mathrm{L}$, low albumin of $26 \mathrm{~g} / \mathrm{L}$, and a strongly positive troponin I of $1.223 \mathrm{ng} / \mathrm{mL}$ (normal range: $<0.05 \mathrm{ng} / \mathrm{mL}$ ). A chest radiograph showed bilateral pleural effusions, perihilar consolidation and a large cardiac silhouette (Fig 2).

\section{Diagnosis and initial management}

The admitting medical team made a diagnosis of acute coronary syndrome (ACS) complicated by AF and a severe communityacquired pneumonia. The patient was commenced on intravenous

Authors: ${ }^{\text {A }}$ infectious diseases registrar, Northwick Park Hospital, Harrow, UK; ${ }^{\text {B }}$ specialty trainee year 5 in cardiology, Kings College Hospital NHS Foundation Trust, UK; ' Consultant cardiologist, Western Sussex Hospitals NHS Foundation Trust, Worthing, UK

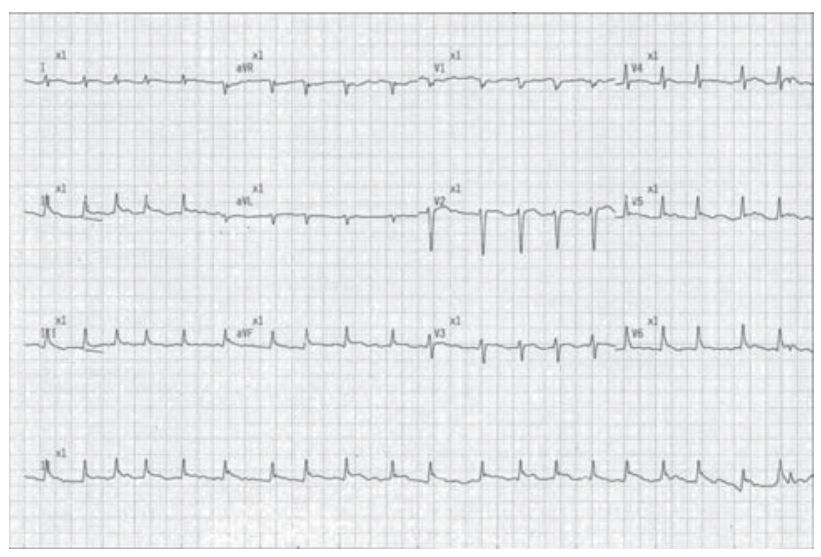

Fig 1. 12-lead electrocardiogram showing atrial fibrillation with a rapid ventricular response, with subtle widespread ST segment elevation with ST segment depression in leads aVR and V1.

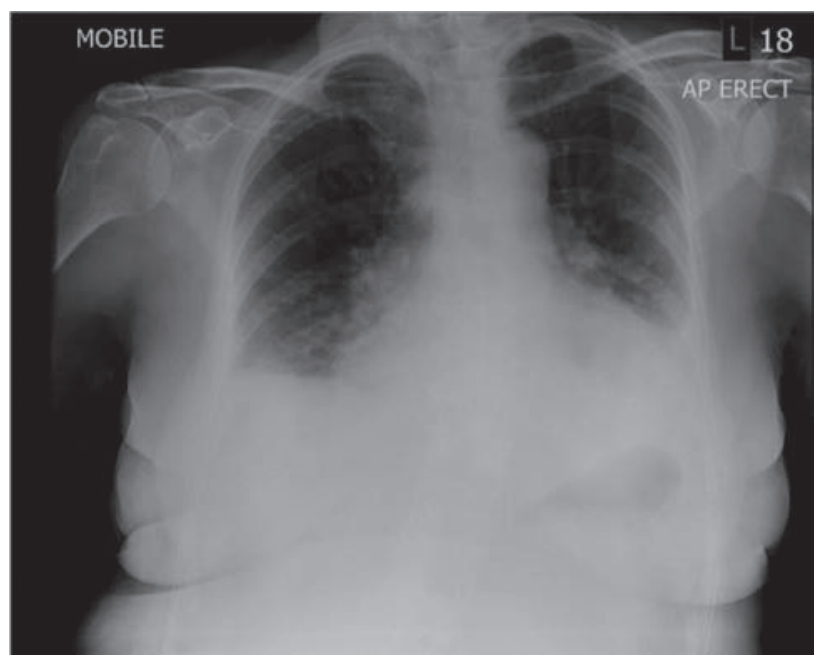

Fig 2. Chest radiograph showing bilateral pleural effusions with perihilar consolidation and a large cardiac silhouette. 
antibiotics (benzylpenicillin and doxycycline), in addition to aspirin, ticagrelor and fondaparinux for ACS. Ventricular rate control with beta blockers was unsuccessful. Several attempts at direct current cardioversion (DCCV) were unsuccessful.

On day 2, the patient was transferred to the coronary care unit under the on-call cardiology team, who felt that the patient did not have an ACS. A diagnosis of sepsis with acute myopericarditis complicated by AF and features of congestive cardiac failure was made. The patient was also noted to be confused and dysarthric. There were no clinical features of tamponade. There was no history of headache or neck stiffness and there were no peripheral stigmata of endocarditis. A bedside transthoracic echocardiogram confirmed a large circumferential pericardial effusion, with largest diameter $2.95 \mathrm{~cm}$ (Fig 3), without echocardiographic features of tamponade, global mildly impaired left ventricular systolic function with estimated ejection fraction $45-50 \%$ and no significant valvular disease. A computed tomography (CT) head scan was normal. Given the concerns over thromboembolism in AF, the patient was cautiously anticoagulated with intravenous unfractionated heparin and ACS treatment was stopped. Beta blockers, digoxin and cautious intravenous furosemide was administered.

Later that day, admission blood cultures were reported to be growing a Gram-positive diplococcus, which was identified as a potential streptococcus species that was sensitive to benzylpenicillin and awaiting further identification. The differential was therefore widened to include endocarditis, myocarditis, reactive pericardial effusion or pyogenic myopericarditis. Later that day, the patient was transferred to the intensive care unit for supportive management of worsening severe sepsis with systemic inflammatory response syndrome. She did not require blood pressure support. On day 3 , the organism in blood cultures was identified as the Gram-negative diplococcus Neisseria meningitides rather than a streptococcus species. Polymerase chain reaction (PCR) subtyping confirmed $N$ meningitidis, serogroup W135, type 2a. A presumptive diagnosis of myopericarditis was made in light of the crossover features of both myocarditis and pericarditis demonstrated by this clinical syndrome. The features of myocarditis included left ventricular systolic impairment with a significantly elevated troponin and inflammatory markers. ${ }^{1}$ The features of pericarditis included a clinical history compatible with this condition, typical saddle-

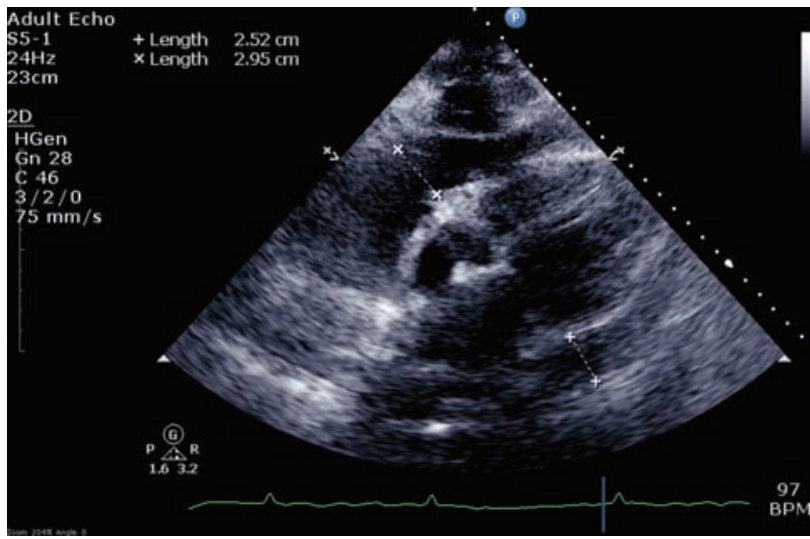

Fig 3. Transthoracic echocardiogram (subcoastal view) demonstrating extensive circumferential pericardial effusion with a widest diameter of $2.95 \mathrm{~cm}$. shaped ST elevation on electrocardiogram (ECG) and the large pericardial effusion identified on echocardiography.

Given the patient's deteriorating clinical state from severe sepsis, worsening gas exchange with bilateral pleural effusions and inability to control her ventricular rate in $\mathrm{AF}$, the patient underwent emergency pericardiocentesis on day 7 to improve her haemodynamic stability and this resulted in immediate symptomatic improvement. The opening pericardial pressure was very high with a mean of $13 \mathrm{mmHg}$ with a prominent ' $y$ ' descent. Following aspiration of $800 \mathrm{~mL}$ straw-coloured fluid, the closing mean pericardial pressure was reduced to $5 \mathrm{mmHg}$ with a normal respiratory pattern. The pericardial aspirate showed inflammatory cells and was PCR-positive for $N$ meningitidis but culture negative. Cerebrospinal fluid was normal and both culture and PCR were negative. Cardiac magnetic resonance imaging was not tolerated due to claustrophobia. Endomyocardial biopsy was not performed.

\section{Progress}

Despite completion of 14 days of benzylpenicillin and considerable clinical improvement, the patient continued to experience persistent troublesome pleuropericarditic chest pains associated with a swinging fever, persistently elevated inflammatory markers and bilateral pleural effusions with a small pericardial effusion. Tuberculosis (TB) was considered but pericardial and pleural aspirates were negative for acid fast bacilli (AFB), and in the context of rapid clinic improvement with antimicrobial therapy and supportive care for sepsis, alongside a low epidemiological risk for TB, this diagnosis was excluded. Autoimmune blood profile and a connective tissue disease screen were negative. Given the well-documented phenomenon in which immune complex disease complicates recovery from meningococcal sepsis, a trial of anti-inflammatory treatment was commenced. There was no response to a course of oral colchicine, but the patient subsequently responded rapidly to a course of oral prednisolone with normalisation of her inflammatory markers and no further fevers. Convalescent transthoracic echocardiography was normal, with resolution of left ventricular systolic function. Cardiac catheterisation showed normal coronary arteries with normal left and right heart pressures. After discharge, the patient was troubled by symptomatic paroxysmal AF and underwent successful pulmonary vein isolation / AF ablation before returning to normal life with no long-term sequelae.

\section{Discussion}

Myopericarditis associated with meningococcal disease is a rare phenomenon. A review of 2091 confirmed cases of $N$ meningitidis infection revealed six cases $(0.3 \%)$ of direct meningococcal pericardial involvement and a further seven cases $(0.3 \%)$ of sterile pericarditis in the context of meningococcal infection. ${ }^{2}$ Three distinct clinical entities are described. ${ }^{3-5}$ Primary meningococcal pericarditis is defined as purulent pericarditis caused by $\mathrm{N}$ meningitidis without evidence of bloodstream infection or meningitis. Disseminated meningococcal disease with pericarditis is caused by early haematogenous spread to the pericardium with subsequent pericardial infection. Reactive (immunopathic) meningococcal pericarditis is a hypersensitivity reaction mediated by immune complexes occurring later in the disease course, often in convalescence. Reactive immune complex complications occurred in 20/130 (15.3\%) of $N$ meningitidis infections in children, 
predominantly manifesting as arthritis, vasculitis and pleuritis. ${ }^{6}$ Invasive disease caused by the W135 serogroup of $N$ meningitidis has increased in prevalence since the year 2000 and its spread worldwide has been associated with an initial outbreak during the Hajj to Mecca. ${ }^{7,8}$ Classically, $N$ meningitidis serogroup C was most frequently associated with pericarditis. ${ }^{9}$ Recently, $N$ meningitidis serogroup W135 has been described as the most predominant strain of meningococcal disease other than meningococcaemia and meningitis, perhaps reflecting its predominant epidemiology at the time of the study. ${ }^{2}$

There is a wide-ranging differential diagnosis for myopericarditis although an underlying diagnosis is often not reached. Causes of myopericarditis include: idiopathic (incorporating viral, post-viral and immune-mediated aetiologies), other infectious aetiologies including tuberculosis, auto-inflammatory causes, malignancy, post myocardial infarction, metabolic causes and drug reactions.

In this case, the patient's history of chest pain and positive cardiac biomarkers led to an incorrect diagnosis of ACS and to the commencement of dual antiplatelet therapy and anticoagulation with the Factor Xa inhibitor, fondaparinux. The presence of atypical chest pain (which is often severe, sharp and postural), febrile illness, tachycardia and saddle-shaped ST elevation are more in keeping with acute pericarditis, and anticoagulation in this scenario can be detrimental. The rise in cardiac biomarkers in this case would have been more in keeping with a non-coronary cause of acute myocardial injury. The causes are probably multiple: the onset of AF with a rapid ventricular response, sepsis, pulmonary oedema or direct involvement of the myocardium.

The clinical priorities in a patient with suspected myopericarditis are to stabilise the patient, to ensure adequate pain control, to assess and treat the underlying aetiology and to assess the extent of underlying cardiac disease. The majority of patients will have a benign clinical course and can be managed conservatively with non-steroidal anti-inflammatories or colchicine. It is imperative to identify those patients who have developed a pericardial collection with impending cardiac tamponade and to seek an urgent cardiology opinion. The assessment should include a detailed history and clinical examination, with assessment for haemodynamic compromise (diaphoresis, tachycardia, shock, raised jugular venous pressure with loss of ' $y$ ' descent and pulsus paradoxus, which is an abnormally large decline in systemic arterial pressure $[>10 \mathrm{mmHg}]$ during inspiration), electrocardiogram, chest radiograph, echocardiogram, basic laboratory tests (full blood count, inflammatory markers, electrolytes, troponin, coagulation screen, blood cultures and HIV serology), as well as consideration of cross-sectional imaging and selected tests for underlying aetiologies. Emergency pericardiocentesis should be performed in cardiac tamponade to restore haemodynamic stability. In other cases, the decision to sample the pericardial fluid must be taken by a cardiologist, considering the extent of haemodynamic compromise from the effusion and the need for diagnostic aspiration. If pericardial fluid is sampled the fluid should be sent for microscopy and culture, including testing for TB and assessment of cytology. In this case, a decision to perform pericardiocentesis was made because of persistent sepsis, pulmonary oedema, pleural effusions and an inability to control the patient's tachycardia in AF. The high pericardial pressure and prominent ' $y$ ' descent would be in keeping with an effusive-constrictive picture.

This case demonstrates both direct $N$ meningitidis myopericardial involvement in the context of a disseminated meningococcal disease and a subsequent protracted steroidresponsive post-infective inflammatory syndrome. It therefore demonstrates features that cross over between the previously described pericardial manifestations of this infection, which to our knowledge has not been reported elsewhere in the literature.

\section{Learning points}

> Neisseria meningitidis infection rarely causes myopericardial disease

> The pathophysiology of meningococcal pericarditis can involve direct meningococcal pericardial infection or reactive phenomena mediated by immune complexes

> Treatment is centered around appropriate antibiotic therapy and supportive measures

> Reactive (immunopathic) meningococcal pericarditis may be steroid responsive

> Raised troponin should be considered in a complete clinical context to avoid inappropriate prescription of dual antiplatelet therapy and anticoagulation.

\section{Consent}

Consent was obtained from the patient to publish the clinical details and images from her case.

\section{References}

1 Caforio AL, Pankuweit S, Arbustini E et al. Current state of knowledge on aetiology, diagnosis, management, and therapy of myocarditis: a position statement of the European Society of Cardiology Working Group on Myocardial and Pericardial Diseases. Eur Heart ] 2013;34:2636-48, 48a-48d.

2 Vienne P, Ducos-Galand M, Guiyoule A et al. The role of particular strains of Neisseria meningitidis in meningococcal arthritis, pericarditis, and pneumonia. Clin Infect Dis 2003;37:1639-42.

3 Zeidan A, Tariq S, Faltas B, Urban M, McGrody K. A case of primary meningococcal pericarditis caused by Neisseria meningitidis serotype $\mathrm{Y}$ with rapid evolution into cardiac tamponade. J Gen Intern Med 2008:23:1532-5.

4 Morse JR, Oretsky MI, Hudson JA. Pericarditis as a complication of meningococcal meningitis. Ann Intern Med 1971;74:212-7.

5 Baevsky RH. Primary meningococcal pericarditis. Clin Infect Dis 1999;29:213-5.

6 Goedvolk C, von Rosenstiel IA, Bos A. Immune complex associated complications in the subacute phase of meningococcal disease: incidence and literature review. Arch Dis Child 2003;88:927-30.

7 Taha MK, Achtman M, Alonso JM et al. Serogroup W135 meningococcal disease in Hajj pilgrims. Lancet 2000;356:2159.

8 Jairam RL, Abdullah MA-R, Rana H et al. Serogroup W-135 meningococcal disease during the Hajj, 2000. Emerg Infect Dis 2003;9:665.

9 Finkelstein Y, Adler Y, Nussinovitch M, Varsano I, Amir J. A new classification for pericarditis associated with meningococcal infection. Eur J Pediatr 1997;156:585-8.

Address for correspondence: Dr Alex J Keeley, Infectious Diseases, Northwick Park Hospital, Watford Road, Harrow HA1 3UJ, UK.

Email: alex.keeley@gmail.com 